\title{
An IoT Solution for Reliable Internet-Based Services
}

\author{
Nalinee Sophatsathit \\ Faculty of Science, Suan Sunandha Rajabhat University, Bangkok, Thailand \\ Email: tnalinee@gmail.com
}

How to cite this paper: Sophatsathit, N. (2018) An IoT Solution for Reliable Internet-Based Services. Journal of Software Engineering and Applications, 11, 129-137. https://doi.org/10.4236/jsea.2018.113008

Received: February 19, 2018

Accepted: March 25, 2018

Published: March 28, 2018

Copyright $\odot 2018$ by author and Scientific Research Publishing Inc. This work is licensed under the Creative Commons Attribution International License (CC BY 4.0).

http://creativecommons.org/licenses/by/4.0/

\begin{abstract}
This paper presents a straightforward solution to an existing internet service so as to enhance its reliability and safety. As the internet society proliferates, myriad of applications, services, and devices are added. Variations among these "things" become unmanageably incompatible. The advent of the Internet of Things or IoT has shed some light on the interoperability issue of these hodgepodges. The proposed framework thus rests on these IoT to achieve such objectives without having to reinvent the wheel or resort to any complex cost-ridden devices and software to operate. The principle is to set up an activity framework that integrates necessary devices to support the designated functionality. This in turn runs on mobile network to serve the internet community. A case study on taxi service apps, having CCTV, GPS, and other monitoring devices and software, is conducted to gauge the viability of the proposed framework. The survey result shows that users are concerned with a few reliability and IoT connectivity factors, namely, cost, schedule, delay, number of vehicles and their proximity. The prospect is promising to broaden the proposed framework in other means of transportation.
\end{abstract}

\section{Keywords}

IoT, Reliable Service, Interoperability, Mobile Network, Smart Phone

\section{Introduction}

The advent of the Internet has brought about myriad of information connectivity in many forms one can imagine. What follows are the supporting methods, techniques, and devices to keep the processing going. As devices are specifically designed to handle different forms of information, their compatibility drifts apart. Middleware, converters, and the likes must be inserted into the connectivity to maintain the information exchange. These phenomena trigger an unprec- 
edented information explosion that creates the infamous social network and face-down society. As people have more information sources to explore, filtering is inevitably called for to aid in making an appropriate choice for oneself. In addition, access mechanisms are handled by a number of physical communication devices such as smartphones, web portals, land-line phones, faxes, and communication protocols, etc. More equipment is added to the network for greater flexibility and means of applications. At the heart of this internet society lies the service whose procedures have been studied, set up, exercised, refined, adjusted, re-implemented, and put to use. The process repeats as services continue and connectivity complexity increases.

From the user's standpoint, more equipment means more selections of apps, convenience of use, and broader application usability. Unfortunately, the fallacy of this provision translates into difficulty and conflicting communication among devices and apps. Despite attempts to resolve such problems, all devices (hardware) and apps (software) are not smoothly integrated and interoperated. The Internet of Things (IoT) offers a promising solution to the above predicament.

The complications involved in such amalgamation of devices, methods, software, or "things", introduce the inherent incompatibility of operations. This makes, among the most important factors, information exchange difficult from many aspects, namely, massive scaling, security, robustness, openness, interoperability, privacy, human in the loop, etc. As a consequence, a number of endeavors are attempted to smooth out this transition. A case in point is traveling which is one of the popular activities that many people do. There is just too much information for anyone to use it efficiently and effectively.

This article thus proposes an IoT framework for carrying out internet-based services reliably so that users will feel secure in using the service. As existing models often focus on logical and physical configurations of the IoT connectivity such as location, distance, traffic volume, shortest path, optimal time complexity of the algorithmic procedures, ease of application use, response time, etc., there is virtually none that focuses on safety and reliability of service from the users' standpoint. A case study in traveling transportation will be presented to gauge the viability of the proposed framework from users' standpoint that sets itself apart from existing works. A light weight solution is presented which focuses primarily on the user's point of view.

This article is organized as follows. Section 2 will discuss some related prior works on IoT. Section 3 elucidates the proposed framework. A small case study is described in Section 4. Some fine points are further explained with future work in Section 5.

\section{Related Work}

The Internet of things (IoT) is a new technology that provides diversified platforms to serve the users' needs [1], and a system integration tool which connect heterogeneous devices into a network of information systems [2]. An important 
functionality of IoT is linking different things on the internet as a virtual aggregate to serve the users' needs [3]. The exchange of data among these things plays a vital role to warrant smooth and seamless IoT operations, in particular, the issues on massive scaling. The "things" could interact in real-time with other things by using various devices and protocols. Its characteristics were comprehensive beyond general applications since they had no limits of time and place [4]. Guo, et al. [5] presented an IoT framework which was initiated by human to build decentralized ad hoc networks for information sharing. IoT has been used in industrial contexts with the help of Message Queuing Telemetry Transport (MQTT) protocol [6]. Among communication protocols of IoT, MQTT is an OASIS standard of lightweight TCP approach developed by IBM and Eurotech [7]. MQTT is a broker-based protocol to manage publish/subscribe messages from a sender to a receiver using queueing techniques [8]. It was proven by large firms such as Amazon and Facebook [9] owing to handling large message transmissions from resource-constrained devices to resource-rich devices. This was reliably defined by Quality of Service (QoS) to guarantee message transmission without loss under conditions [10], namely, low, medium, and high guarantee levels.

In a nutshell, IoT facilitate people and things to easily work together [1]. Unfortunately, data transmission cost, IoT software cost, human cost, are a few high costs that users must take into account for what it is worth.

Bearing the aforementioned issues in mind, this study will make use of some important aspects of prior work, namely, human in the loop, interoperability, and reliability. The proposed framework will consider these aspects to establish a workable solution. Details will be described in the next section.

\section{Proposed Approach}

The proposed internet-based service exploits the abundant availability of IoT devices to improve service reliability. Figure 1 illustrates a typical internet-based service that rests heavily on human and manual operations. Input data come primarily from web, land-line phone inquiry, and mobile phone access. Processing is carried out manually via human operator conversation and off-line reply. Outputs are transmitted through operator, web site, or central office in the form of brochures, facsimile, etc. Communication exchange is performed via conventional slow media. Although this form of service is considered reliable to some extent, it is too slow to respond in some urgent situations, especially for out of town visitors who need immediate help information.

The proliferation of mobile connection creates an overwhelming information explosion. People have more information sources to choose, thereby information filtering is required to select an appropriate choice. Access mechanisms are handled by a number of physical communication devices such as smart phones, web portals, access points, etc., as shown in Figure 2.

The principal differences to be addressed in this study from the typical internet-based services as shown above are transportation cost, transport schedule, 


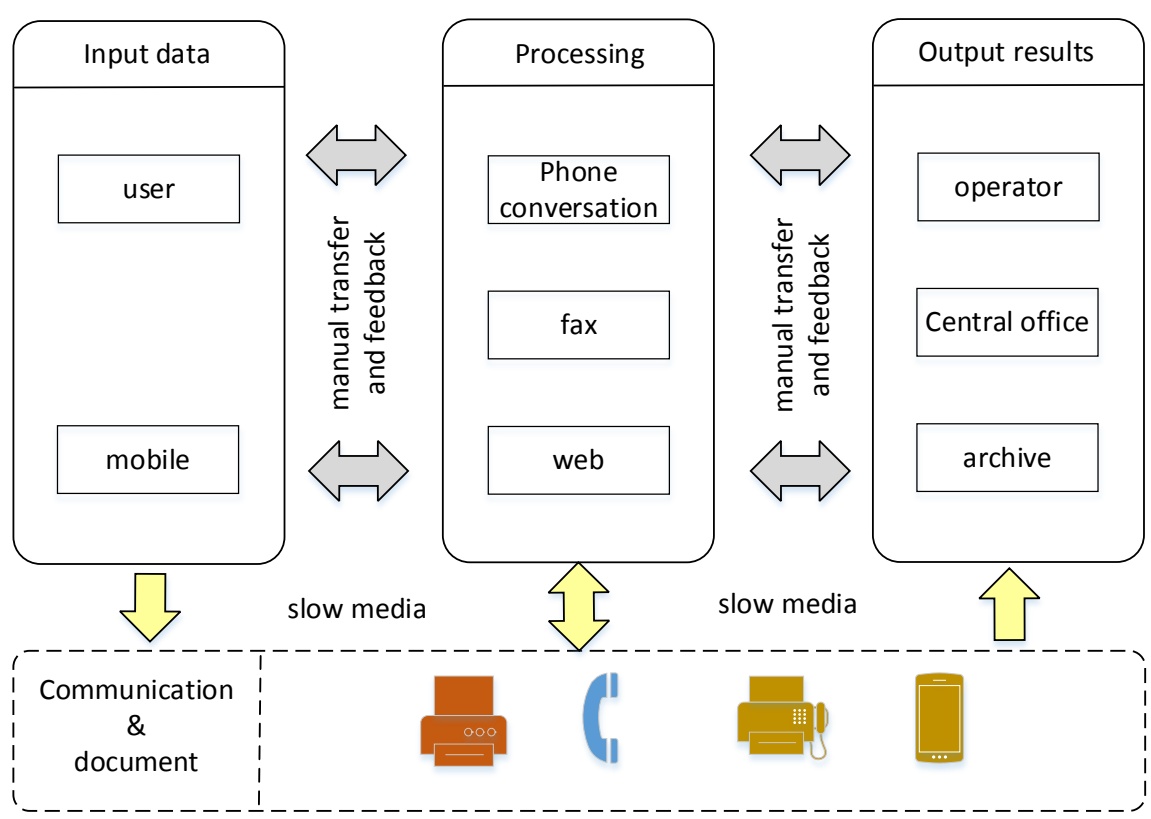

Figure 1. A typical internet-based service.

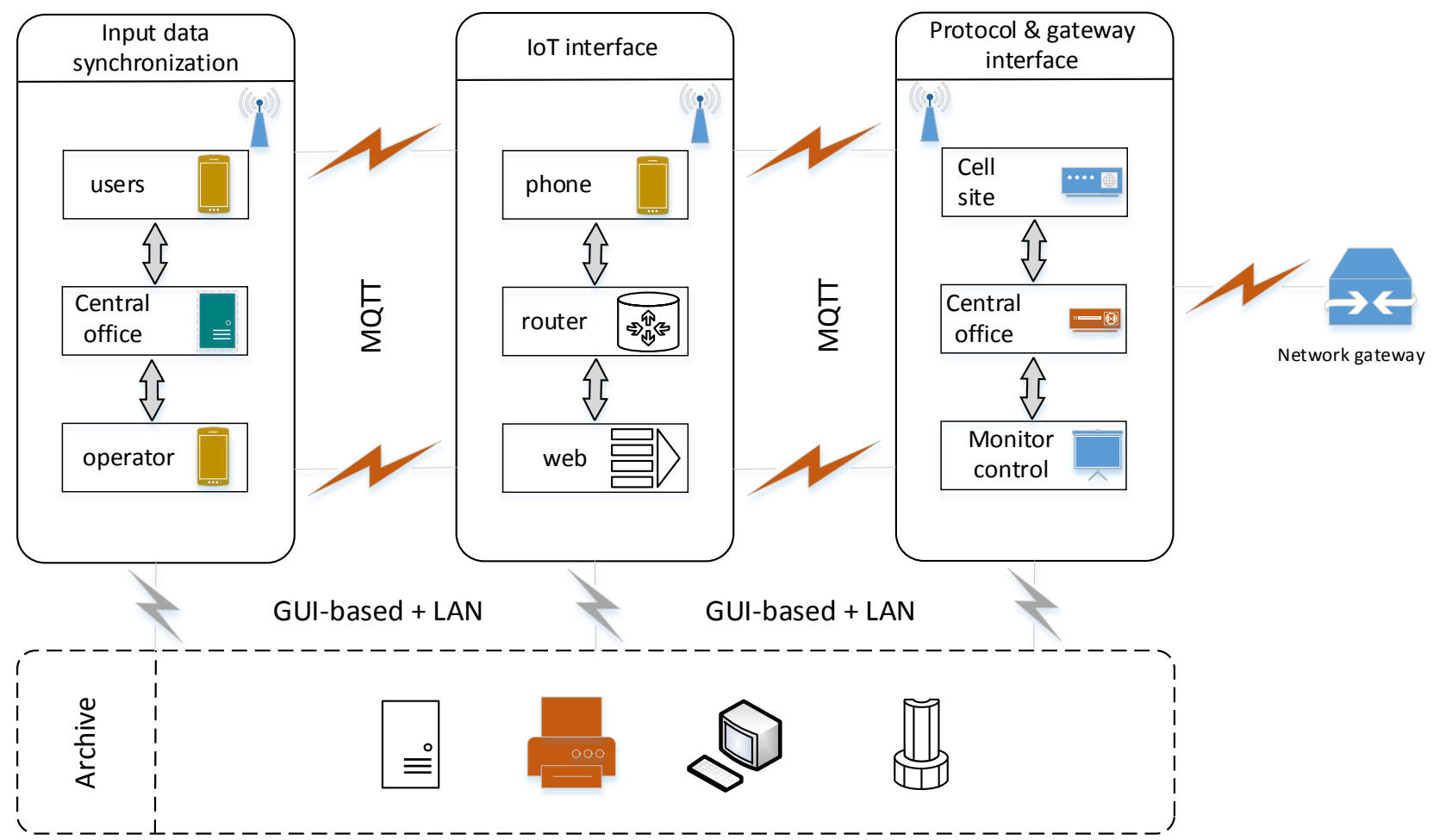

Figure 2. The proposed IoT solution for internet-based service.

and user cancellation. In typical internet-based services, users were concerned about trust worthiness of the service operator (taxi driver) as he could manipulate routing and time of travel. These affected the cost and time spent that, in some cases, could result in missing schedule or appointment on the user's part. Meanwhile, user was unable to cancel the service if it turned out unsatisfactory 
such as waiting for too long. The proposed service framework will explore these issues in detail.

Consider an existing traveling scenario as shown in Figure 3. Passengers (or users) place a request to a dispatch center for a taxi. Three possible cases could happen. First, the operator accepts the request and dispatches a pick up. Second, the operator accepts the request but no taxi takes the pickup. Third, the pickup becomes a no-show in the last minutes. Only the first scenario is a fruitful operation. The other two are dissatisfactory operations that should not happen with any successful business. A better solution that is gaining acceptance is the use of application alert. The taxi driver can reserve a pick up, ignore it, or relinquish his turn in the last permissible interval. In which case, the second stand-by pick up is alert to take over. However, this solution might not satisfactorily workout in some situations such as heavy traffic, accident, opportunity waste of the stand-by taxi, etc.

The proposed approach, on the contrary, allows the passenger to place a request as usual. The apps will only look for registered taxis within a predetermined distance who respond to the pickup alert and sort out the nearest taxi in proximity order. In addition, a few precautions are established to insure operational safety. For instance, CCTV in the taxi compartment, real-time location monitor via GPS tracking, service area coverage, publicly available QR code for ease of taxi access, driver information, transaction log, etc., to insure the passenger themselves and their close ones a peace of mind. All these require interoperating devices over the IoT networking to handle the ride and good service. A hierarchical operation scheme is shown in Figure 4, where four layered applications are conceptually organized to render operational independence between layers. The performance measurement function is placed at the entry and exit points of the operation scheme, whereby some important statistics can be collected and analyzed accordingly.

At the application layer, a user accesses the system via access activity apps. His related information is filtered to compose an inquiry which is subsequently sent

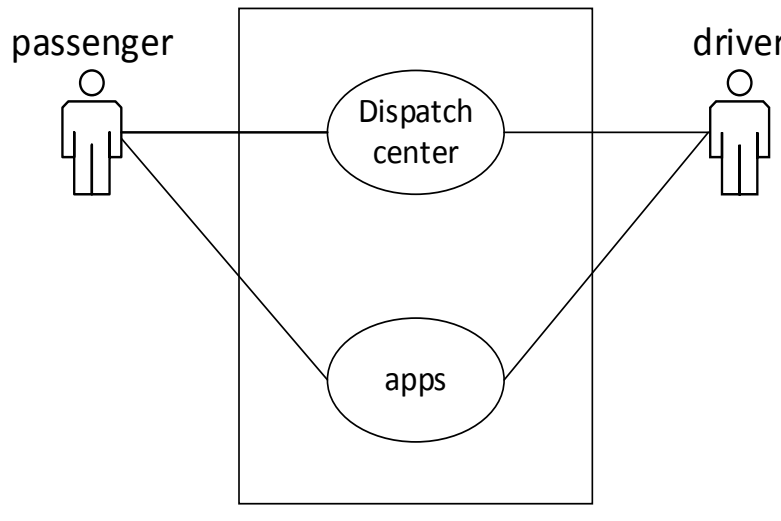

(a)

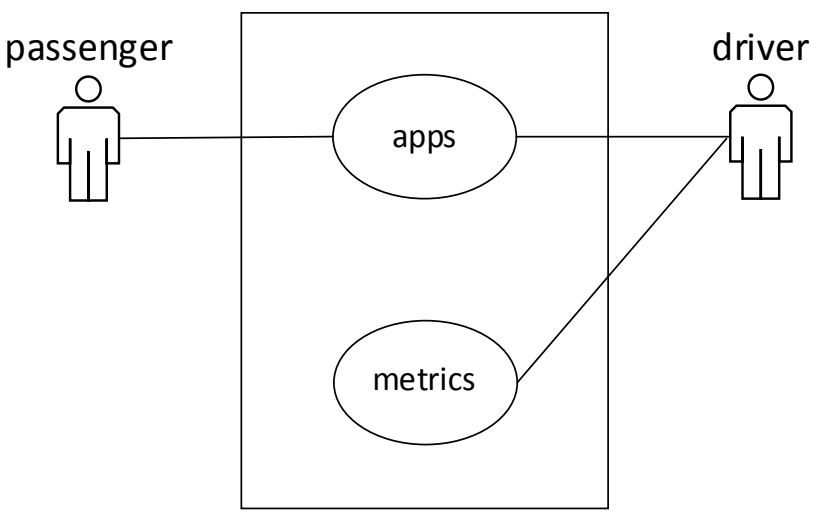

(b)

Figure 3. A case-study of taxi service (a) existing taxi pick up (b) proposed taxi pick up. 


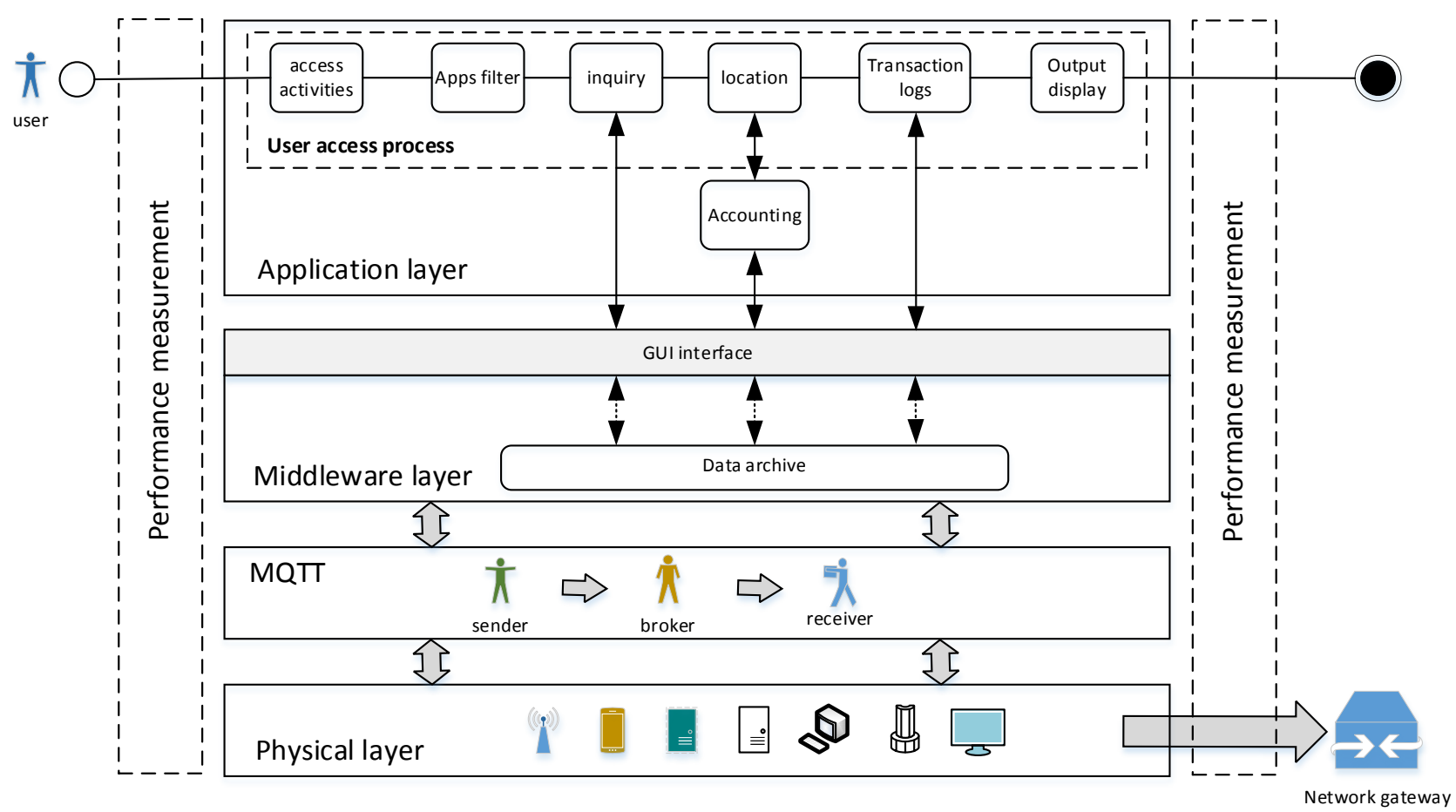

Figure 4. Activities of the proposed system.

through the GUI interface to the middleware layer. Meanwhile, his location information is submitted to accounting module for transaction recording. The output is displayed on the user's device for activity summary.

The middleware layer archives the transactions and proceeds through the MQTT protocol layer. This in turn communicates with physical layer to transmit information out to the network gateway. All these set ups can be configured with existing IoT technologies and devices.

The following performance statistics could be gathered from the above proposed system activities:

1) Number of accepted requests from the passengers

2) Number of registered pickups

3) Number of successful pickups

4) Duration of wait for pickup

5) Average registered pickup

6) Business ratio

The first statistic is a direct request from the passenger. The second one counts the number of taxis within the allowable proximity registered to pick up. This number limits to two to prevent opportunity waste for the stand-by taxi. These two statistics are obtained from input performance measurement. The number of successful pickups counts of the passengers who reach their destination. Duration of wait measures how long the taxi waits for the passenger. The average registered pick up tallies both successful and opportunity waste pickups for future service improvement, that is, no stand-by registered taxi required yet still insure $100 \%$ pick up. The last statistic evaluates how well the request is suc- 
cessfully fulfilled by determining the ratio of number of passengers' pickups to number of requests.

\section{Case Study}

A preliminary survey was conducted on 86 out of 110 respondents (or $78.2 \%$ feedback) to gauge user satisfaction with the above scenarios. All respondents were users of existing taxi service operators who placed their calls over the phone, although a couple of apps were available and gaining popularity. At any rate, the good old phone calls still prevailed. Most of the calls were placed at night since people did not want to go out just to get a taxi. Day time calls were less due to the availability of other public transportation means that were less expensive. In the survey, users were asked with a set of service arrangements based on what was described. The results are shown in Table 1, where the significant factors reflect user satisfaction.

From Table 1, the variance percentage of reliability is higher than that of IoT connectivity since considerations of factors influencing the service are high. Users were concerned with unpredictable cost due to traffic volume and schedule constraint, while IoT devices should provide reasonably reliable outcome. For example, the number of vehicles in picked up queue within 5 - 10 minutes away from the designated user's location, the delay of signal for monitoring current location and passenger presence in car caused by communication network traffic, etc. From implementation standpoint, the apps and real services are underway yet lack the publicity to collect any user feedback. As a consequence, no statistics could be gathered.

One may contend that the case study focuses on very few aspects of internet-based services that seem trivial and are not worthwhile. The fact is that thefts, sexual assaults, and in extreme case murders, occur occasionally with taxi passengers. Recent social pressure not only prompts for the applications of taxi service apps as mentioned earlier, but also ways of monitoring and tracking of passenger safety during the taxi ride. The application of CCTV and GPS devices and their companion software are part of Department of Transportation's mandates to alleviate such predicaments. Studies on one matter of life and death are

Table 1. Factors influencing taxi service.

\begin{tabular}{cl}
\hline Issue & \multicolumn{1}{c}{ Factors } \\
\hline & apps: \\
& $\%$ variance $=21.3$ \\
Reliability & - Transportation cost \\
& $-\quad$ Transport schedule \\
& - User cancellation \\
& metrics: \\
& $\%$ variance $=12.7$ \\
& $-\quad$ Delay \\
& $-\quad$ Number of vehicles in service queue \\
& - proximity \\
\hline
\end{tabular}


more than enough to justify the cost. This study merely proposes a viable framework as an alternative solution to attain such social well-being concerns.

Whatever the results of the case study turned out, one conclusion that could be drawn was the integration of existing devices being exploited to work together under one roof-the Internet of Things.

\section{Conclusion and Future Work}

This study explores the integration of existing devices and technologies to arrive at a working solution for real world problems. The exploitation of IoT renders not only new services, but also reliable and satisfactory solutions without having to reinvent the wheel or resort to any complicated systems. It only takes some observations to select proper and inexpensive solutions. Various devices are incorporated for service reliability and safety such as CCTV, GPS, software support, etc. As a consequence, performance and efficiency measures can be collected, studied, and adjusted to enhance service coverage, tracking, ease of service functionalities (e.g., payment method, advanced reservation).

Future work should look into the impacts on the performance statistics outlined earlier so as to broaden service reliability and IoT connectivity. This will by all means broaden the service bases, such as tandem connections by passenger traveling from airport by train to taxi in reaching home safely. In which case, access activities in the application layer must be revised to accommodate the extension. Moreover, applicability of the proposed system should incorporate more services that encompass public transportation means, regular and chartered operators, etc., as mobile devices become the norm of IoT access to all transportation services such as buses, airplanes, boats, shared bicycles and cars, etc.

\section{References}

[1] Stankovic, J.A. (2014) Research Directions for the Internet of Things. IEEE Internet of Things Journal, 1, 3-9. https://doi.org/10.1109/JIOT.2014.2312291

[2] Li, S.C., Oikonomou, G., Tryfonas, T., Chen, T.M. and Xu, L.D. (2014) A Distributed Consensus Algorithm for Decision Making in Service-Oriented Internet of Things. IEEE Transactions on Industrial Informatics, 10, 1461-1468. https://doi.org/10.1109/TII.2014.2306331

[3] Chen, S., Xu, H., Liu, D., Hu, B. and Wang, H. (2014) A Vision of IoT: Applications, Challenges, and Opportunities with China Perspective. IEEE Internet of Things Journal, 1, 349-359. https://doi.org/10.1109/JIOT.2014.2337336

[4] Weinberg, B.D., Milne, G.R., Andonova, Y.G. and Hajjat, F.M. (2015) Internet of Things: Convenience vs. Privacy and Secrecy. Business Horizons, 58, 615-624. https://doi.org/10.1016/j.bushor.2015.06.005

[5] Guo, B., Zhang, D.Q., Wang, Z., Yu, Z.W. and Zhou, X.S. (2013) Opportunistic IoT: Exploring the Harmonious Interaction between Human and the Internet of Things. Journal of Network and Computer Applications, 36, 1531-1539. https://doi.org/10.1016/j.jnca.2012.12.028

[6] Whitmore, A., Agarwal, A. and Xu, L.D. (2015) The Internet of Things-A Survey 
of Topics and Trends. Information Systems Frontiers, 17, 261-274.

https://doi.org/10.1007/s10796-014-9489-2

[7] Luzuriaga, J.E., Perez, M., Boronat, P., Cano, J.C., Calafate, C. and Manzoni, P. (2016) Improving MQTT Data Delivery in Mobile Scenarios: Results from a Realistic Testbed. Mobile Information Systems, 2016, Article No. 4015625.

[8] Razzaque, M.A., Milojevic-Jevric, M., Marija, A. and Clarke, S. (2016) Middleware for Internet of Things: A Survey. IEEE Internet of Things Journal, 3, 70-95. https://doi.org/10.1109/JIOT.2015.2498900

[9] Hwang, H.C., Park, J. and Shon, J.G. (2016) Design and Implementation of a Reliable Message Transmission System Based on MQTT Protocol in IoT. Wireless Personal Communications, 91, 1765-1777. https://doi.org/10.1007/s11277-016-3398-2

[10] Aziz, B. (2016) A Formal Model and Analysis of an IoT Protocol. Ad Hoc Networks, 36, 49-57. 